\title{
Phase Retrieval and Induction Mapping of Artificially Structured Nanometric Magnetic Arrays
}

\author{
Y. Zhu, V.V. Volkov, M. Schofield, M. Beleggia, J. Lau and M. Malac* \\ Materials Science Department, Brookhaven National Laboratory, Upton, NY 11973, USA \\ *also Department of Physics, University of Alberta, Edmonton, T6G2J1, Canada
}

Understanding nanometer scale magnetization and induction distribution of artificially structured magnetic materials is a subject of intensive research due to the ever decreasing in size of the magnetic elements in recording and storage media [1]. Although off-axis electron holography has played a crucial role in nanoscale characterization of the domain configuration, its applications are often hampered by limited area of view in experiment.

Facing the challenge, recently we have developed a phase retrieval procedure based on the Transport-of-Intensity Equation combined with image alignment, distortion correction and symmetrization algorithms to map local magnetization [2]. The method agrees well with electron holography analysis but is more versatile in length scale. Fig.1 shows a Lorentz image of Permalloy $\left(\mathrm{Ni}_{20} \mathrm{Fe}_{80}\right)$ arrays fabricated using an in-house UHV film deposition system with an artificially structured mask. The induction distribution, derived using the method and shown as a vector color map, directly reveals the complex magnetic structure, its dynamic behavior and low-energy state including fringing field by comparison with micromagnetics simulations [3]. Fig.2 gives another example of artificially structured magnetic Ni dot-array prepared at even smaller dimension using TEM based lithography. The modified phase-gradient image and phase contour of the boxed area in Fig.2a are shown in Fig.2b and c, respectively.

As we know, the measured phase of an objective wave carries information about both the electric and magnetic field distribution in the sample and can be expressed in a two-dimensional case as

$\phi(r)=\phi_{E}+\phi_{M}=C_{E} \oint V(r, z) d z-\frac{e}{\hbar} \iint B(r, z) \cdot d A$

where $z$ and $r$ are position vectors and $d A$ is the area element bounded by the beam path. $C_{E}=6.25 \times 10^{-3} \mathrm{rad} / \mathrm{Vnm}$ for $300 \mathrm{kV}$ electrons, and $V$ and $B$ are electrostatic and magnetic potential, respectively. To quantify the magnetic contribution one must separate it from the electrostatic contribution. If the thickness of the element does not vary, the electrostatic component will be a constant, and if $V_{0}$ is known, $\phi_{E}$ can be accurately calculated for any geometry. Since the ratio $\phi_{M} / \phi_{E} \approx 0.24 B_{0} D_{0} / V_{0}$ ( $V_{0}$ : the mean inner potential, $B_{0}$ : in-plane component of the induction), for $V_{0}=10 \mathrm{~V}$ and $B_{0}=1$ Telsa, the two components have about the same contribution for a particle size of $D_{0} \sim 40 \mathrm{~nm}$, as shown in Fig.3. However, for smaller elements the electrostatic component dominates. When $D_{0}$ reaches to $4 \mathrm{~nm}$ the contribution of the magnetic potential is only $1 / 10$ of the electrostatic counterpart. Thus, quantification of magnetic induction distribution for subnanometer elements imposes a real challenge for modern electron microscopy [4].

[1] Sym. R, Advanced characterization of artificially structured magnetic materials, MRS Fall Meeting 2002.

[2] V.V. Volkov, Y. Zhu and M. De Graef, Micron 33 411-416 (2002).

[3] see the 4 related abstracts (Beleggia et al, Lau et al, Volkov et al, Volkov and Zhu) by the Brookhaven Group in the proceeding.

[4] Work supported under US DOE contract No. DE-AC02-98CH10886. 

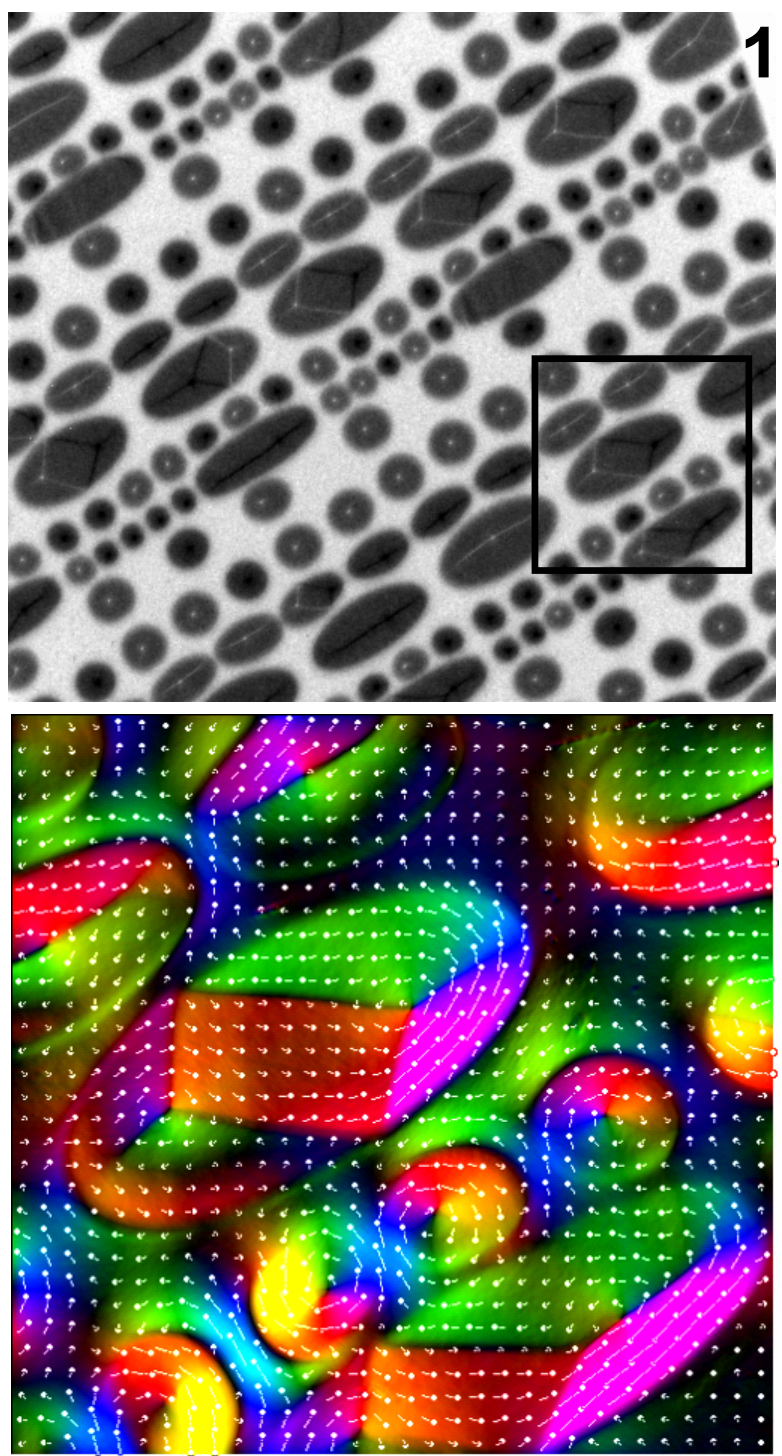

Fig.1 Lorentz image and projected induction distribution (boxed area) of the artificially patterned $\mathrm{Ni}_{20} \mathrm{Fe}_{80}$ Permalloy. The diameter of the small dots are about 1 um.

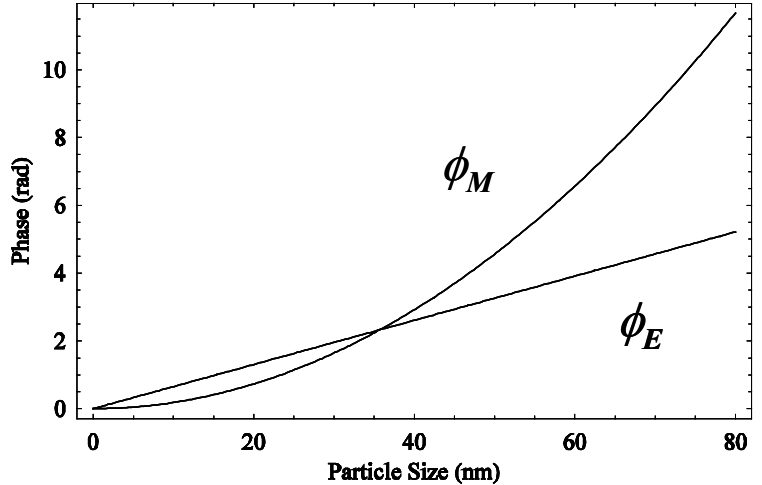

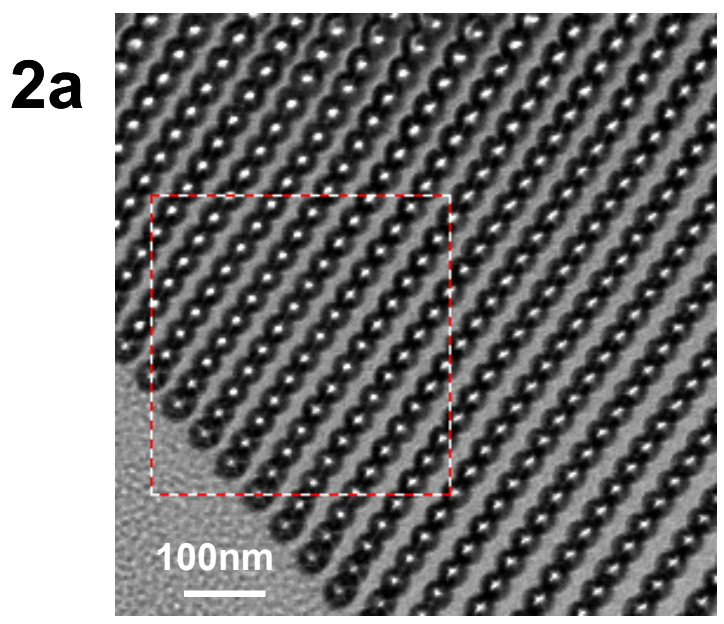
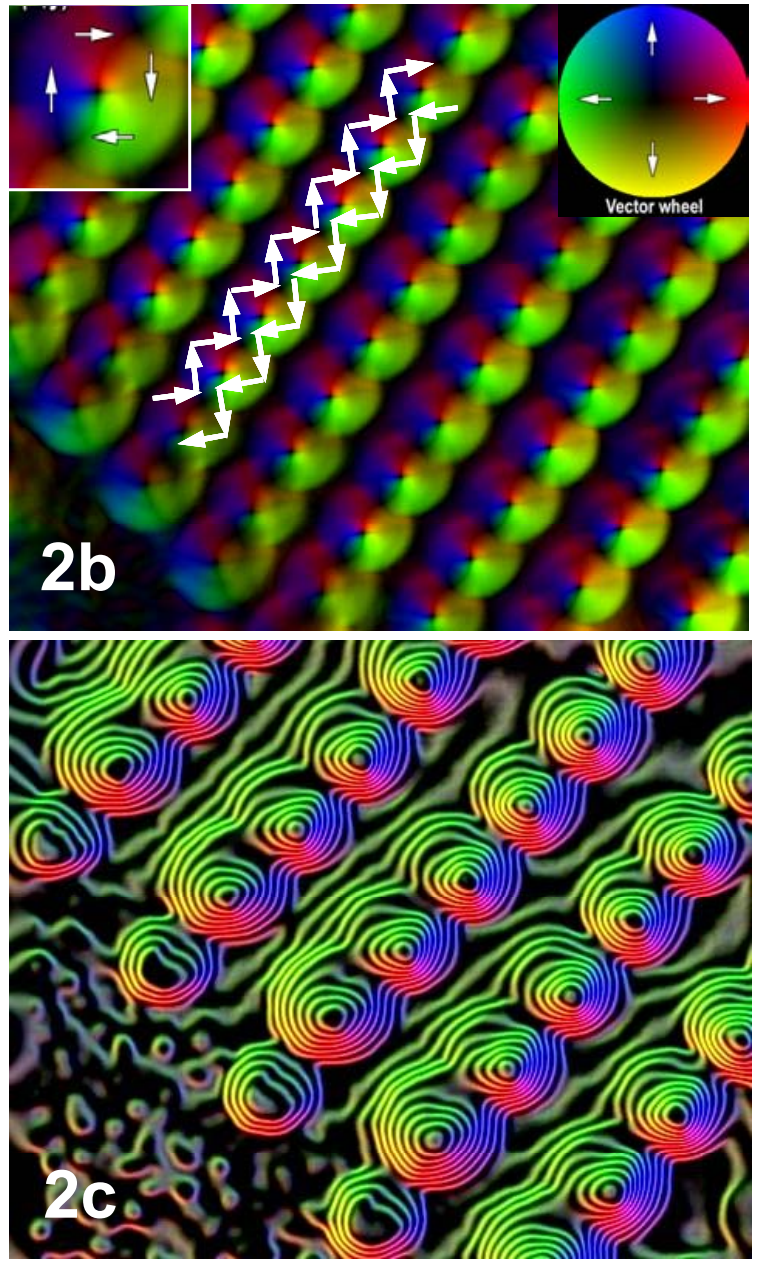

Fig.2 Ni dot array (40nm in diateter). (a) Lorentz image, (b) phase-gradient image, and (c) phase contour.

Fig.3 Magnetostatic and electrostatic contribution to the total phase as a function of particle size. 